\title{
MÉTODO DE AVALIAÇÃO DO COEFICIENTE DE ATRITO ENTRE MOLDE E VEIO EM MÁQUINAS DE LINGOTAMENTO CONTÍNUO DE PLACAS*
}

\author{
Franz Ramstorfer ${ }^{1}$ \\ Eduardo Manzano ${ }^{2}$ \\ Márcio Paulino Ferreira ${ }^{3}$
}

\section{Resumo}

No lingotamento contínuo de placas, rompimentos de veio pelo colamento e problemas de qualidade de superfície, como trincas longitudinais, são frequentemente relacionadas à lubrificação insuficiente no molde de lingotamento contínuo devido ao pó fluxante ou a prática de oscilação inadequados. Para detectar, com vistas a prevenir, este tipo de problema de processo, um método para avaliar em tempo real o coeficiente de atrito entre o veio e as placas do molde foi desenvolvido, utilizando-se a medição da força que atua nas placas laterais do molde e as forças de oscilação. Observou-se que o coeficiente de atrito do molde é um parâmetro confiável para auxiliar no desenvolvimento de pó fluxante, na otimização de parâmetros de oscilação e no monitoramento online do desempenho de pó fluxante.

Palavras-chave: Molde de lingotamento contínuo; Coeficiente de atrito do molde.

\section{METHOD OF EVALUATION OF FRICTION COEFFICIENT BETWEEN MOLD AND STRAND IN CONTINUOUS SLAB CASTING MACHINES}

\begin{abstract}
In continuous casting of slabs, sticker breakouts and surface quality problems, like longitudinal cracks, are often related to insufficient lubrication in the continuous casting mold due to an inappropriate casting powder or oscillation practice. In order to detect this type of process problem, a method to evaluate in real time the friction coefficient between the strand and the mold plates was developed using the measurement of the force acting on the lateral mold plates and the oscillation forces. It was observed that the mold friction coefficient is a reliable parameter to assist in the development of mold powder, in the optimization of oscillation parameters and in the online monitoring of mold powder performance.
\end{abstract}

Keywords: Continuous casting mold; Mold friction coefficient.

Engenheiro Mecânico, Ph.D., Especialista de processos, Usiminas, Cubatão, SP, Brasil.

Engenheiro Mecânico, Coordenador de manutenção, Usiminas, Cubatão, SP, Brasil.

Engenheiro Eletricista, Especialista de manutenção, Usiminas, Cubatão, SP, Brasil. 


\section{INTRODUÇÃO}

No lingotamento contínuo de placas, vários defeitos de superfície do veio e rompimentos de veio são originários dos processos dentro do molde como a transferência de calor e o atrito entre o veio e as placas de molde oscilantes. $O$ atrito é influenciado principalmente pelas propriedades do pó fluxante e os parâmetros da oscilação periódica do molde. Uma visão geral do atrito de molde e suas diversas influências foram mostradas por Wolf [1]. Várias simulações numéricas complexas [2,3] foram conduzidas até agora para melhorar o entendimento dos processos dentro do molde. Também foram desenvolvidos modelos analíticos para prever o atrito de molde. Royzman [4] estudou o modelamento do coeficiente de atrito da parte superior do molde. Suzuki et al. [5] analisaram a tensão de atrito de molde através de um modelo matemático da infiltração do pó fluxante líquido. Aparatos experimentais, que simulavam uma máquina de lingotamento contínuo, foram utilizados por Tsutsumi et al. [6] e Nakato et al. [7] para medir a força de atrito. A força de atrito entre molde e veio foi utilizada para a determinação do comportamento de lubrificação do pó fluxante [7] e o ajuste da prática de oscilação $[5,8]$.

Hoje em dia, a determinação da força de atrito em tempo real por meio de aplicação de células de carga ou transdutores de pressão ao molde $[9,10]$ é estado da arte e largamente implementada, como na máquina de lingotamento 3 em Cubatão. Nesta máquina, a tensão de atrito é determinada através do processamento digital em tempo real da posição do oscilador e a pressão hidráulica nos cilindros do oscilador durante um curso de trabalho. A figura 1a mostra a força e o movimento de oscilação em função do tempo, e a figura $1 \mathrm{~b}$ mostra estes valores em um processo cíclico. A área dentro deste processo cíclico conforme a figura $1 \mathrm{~b}$ é conhecida como "trabalho a quente" [Joule], sendo o trabalho que o oscilador tem que fornecer durante lingotamento. A força de atrito média é determinada através da divisão do trabalho de atrito efetivo pelo curso da oscilação, como mostrado na figura 1c. $\mathrm{O}$ trabalho de atrito efetivo é o trabalho a quente subtraído pelo trabalho a frio (trabalho de atrito sem veio dentro do molde). A divisão da força de atrito média pela área de contato entre molde e veio (circunferência do molde multiplicado com o comprimento do molde efetivo) resulta em um valor da tensão de atrito.

(a)

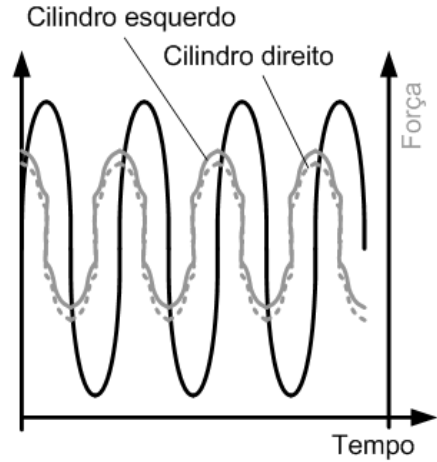

(b)

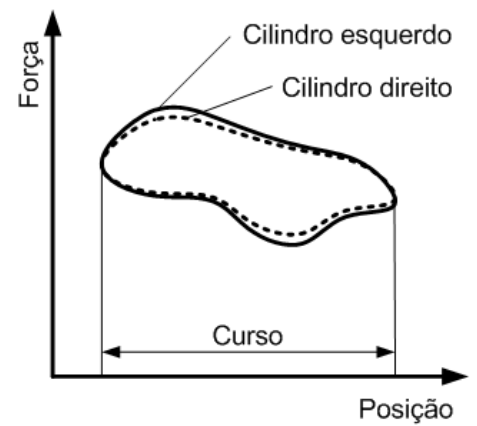

(c)

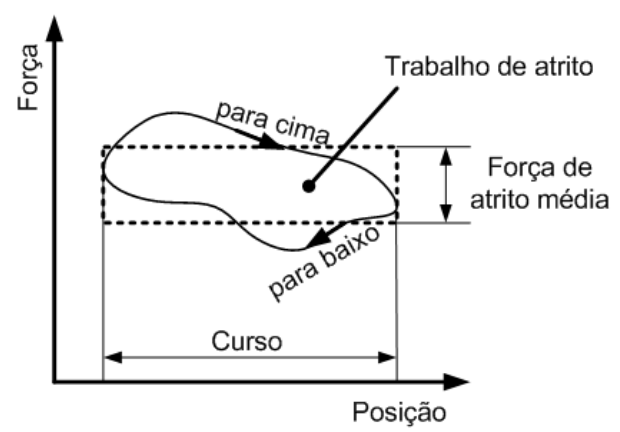

Figura 1. Movimento e força de oscilação; determinação da força de atrito média através do trabalho de atrito.

Na máquina de lingotamento 3 , este cálculo é realizado em tempo real no sistema de monitoramento do molde. A tensão de atrito varia, dependendo das dimensões da placa e as condições de lubrificação, entre 10 e $40 \mathrm{kN} / \mathrm{m}^{2}$. Baseado neste 
monitoramento, especialistas de lingotamento já podem detectar alguns problemas de processo, mas o valor da tensão depende das dimensões da placa, velocidade de lingotamento, condições de fundição do pó fluxante, propriedades do aço, propriedades do pó fluxante entre outros. Uma interpretação ou determinação dos problemas do processo ou qualidade do produto através da tensão de atrito não é fácil, ou às vezes impossível, pois falta um valor de referência.

O objetivo deste trabalho é a determinação do coeficiente de atrito durante o lingotamento em tempo real, para estabelecer um valor independente para avaliar o estado da lubrificação dentro do molde. Wolf [1] utilizou os dados da força de atrito de Suzuki et al. [5] e Nakato et al. [7] para calcular o coeficiente de atrito através da força ferrostática como um valor de força normal. Conforme a figura $2 a$, o coeficiente de atrito situa-se entre 0,15 e 0,25 utilizando os dados de Suzuki et al. [5]. Valores do coeficiente de atrito entre 0,3 e 0,70 foram obtidos por Wolf [1] com a utilização dos dados de Nakato et al. [7], mostrado na figura $2 b$.

(a)

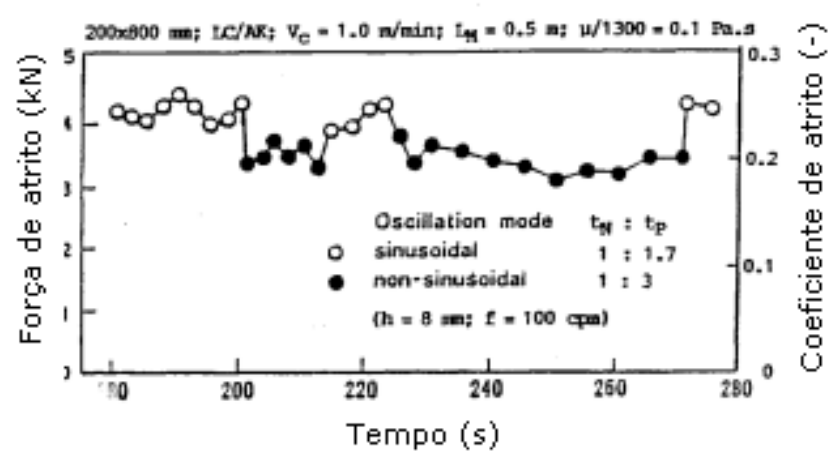

(b)

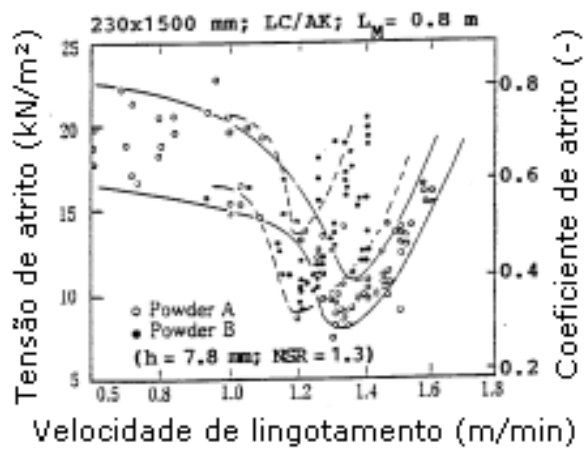

Figura 2. Coeficiente de atrito determinado por Wolf [1].

\section{MATERIAIS E MÉTODOS}

Moldes de lingotamento modernos são também equipados com um sistema de troca de largura hidráulico, proporcionando uma possibilidade simples da determinação das forças atuando nas faces estreitas do molde em tempo real. No molde da máquina de lingotamento 3 de Cubatão por exemplo, basta instalar sensores de pressão aos cilindros hidráulicos, como mostrado na figura 3. Este método já é conhecido, mas devido à falta dos benefícios práticos desta informação adicional, ele não está sendo aplicado amplamente.

Para determinar o coeficiente de atrito entre as placas do molde e o veio, foram combinados os dois métodos descritos anteriormente, a medição da tensão de atrito e a medição das forças atuando nas placas laterais do molde.

Como primeiro passo, os valores médios das forças de ambos os cilindros inferiores $\left(F_{\text {cil_inf_dir, }} F_{\text {cil_inf_esq }}\right)$ e superiores $\left(F_{\text {cil_sup_dir, }} F_{\text {cil_sup_esq }}\right)$ são calculados:

$$
\begin{aligned}
& \mathrm{F}_{\text {cil_inf }}=\left(\mathrm{F}_{\text {cil_inf_exq }}+\mathrm{F}_{\text {cil_inf_dir }}\right) / 2 \\
& \mathrm{~F}_{\text {cil_sup }}=\left(\mathrm{F}_{\text {cil_sup_esq }}+\mathrm{F}_{\text {cil_sup_dir }}\right) / 2
\end{aligned}
$$

Neste cálculo foi assumida uma distribuição de força especifica linear $q_{\text {estr }}(z)$ ao longo do comprimento do molde L, como mostrado na figura 4. 


\section{$46^{\circ}$}

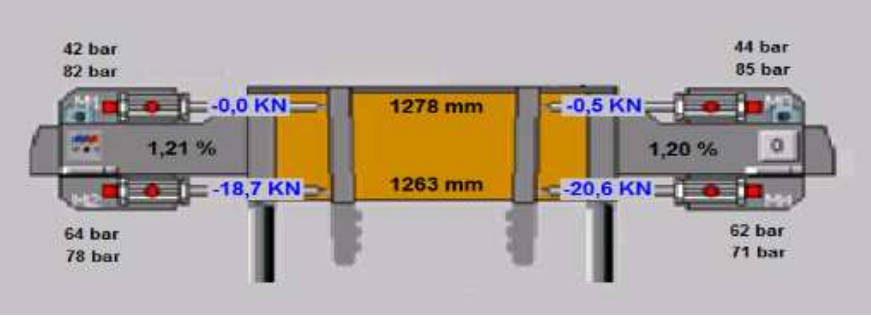

Figura 3. Tela do Nível 1 mostrando a medição das forças atuando nas placas laterais do molde.

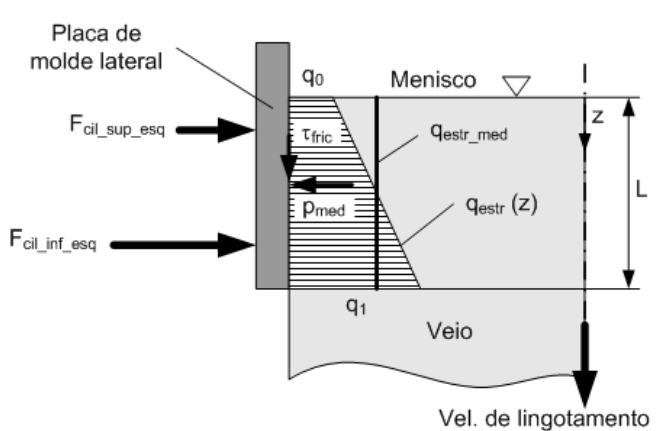

Figura 4. Forças atuando na placa de molde lateral.

Conforme a figura 4, o equilíbrio de força pode ser escrito como:

$$
\mathrm{F}_{\text {cil_inf }}+\mathrm{F}_{\text {cil_sup }}=\int_{0}^{\mathrm{L}} \mathrm{q}_{\text {est }}(\mathrm{z}) \cdot \mathrm{dz}=\int_{0}^{\mathrm{L}}\left(\mathrm{q}_{0}+\frac{\mathrm{q}_{1}-\mathrm{q}_{0}}{\mathrm{~L}} \cdot \mathrm{z}\right) \cdot \mathrm{dz}=\frac{\mathrm{q}_{0}+\mathrm{q}_{1}}{2} \cdot \mathrm{L}=\mathrm{q}_{\text {estr_med }} \cdot \mathrm{L}
$$

A força especifica média qestr_med é então dividida pela espessura do veio $T$ das placas laterais, resultando em uma pressão normal média $p_{m e d}\left[\mathrm{kN} / \mathrm{m}^{2}\right]$ que atua nas faces laterais:

$$
\mathrm{p}_{\mathrm{med}}=\frac{\mathrm{q}_{\text {estr_med }}}{\mathrm{T}}=\frac{\left(\mathrm{F}_{\text {cil_inf }}+\mathrm{F}_{\text {cil_sup }}\right)}{\mathrm{L} \cdot \mathrm{T}}
$$

O coeficiente de atrito, $\mu_{\text {fric }}$, é determinado, de acordo com a lei de atrito [11], através da divisão da tensão de atrito $\tau_{\text {fric }}$ pela pressão normal média $p_{\text {med: }}$

$$
\mu_{\text {fric }}=\frac{\tau_{\text {fric }}}{p_{\text {med }}}
$$

\section{RESULTADOS E DISCUSSÃO}

O sistema de monitoramento do coeficiente de atrito em tempo real foi implementado na máquina de lingotamento 3 em Cubatão. Um valor típico do coeficiente de atrito $c_{\text {fric }}$ foi observado entre 0,25 e 0,35, que se correlaciona bem com os valores apresentados por Wolf [1].

Através do monitoramento do coeficiente de atrito do molde em tempo real, problemas do processo e da qualidade do produto podem ser detectados, pois o coeficiente de atrito permite, ao contrário da tensão de atrito, uma comparação entre as várias dimensões da placa, tipos de aço e as velocidades de lingotamento. Essa independência do coeficiente de atrito é demonstrada nas próximas figuras 5 e 6 .

Em um exemplo prático conforme a figura $5 \mathrm{a}$, a força inferior atuando em uma placa lateral e o trabalho a quente mudam durante um período de uma maneira síncrona, ao contrário do coeficiente de atrito, calculado através destes dados conforme equação (5), que não muda significativamente durante o mesmo período conforme a figura $5 b$.

A mesma observação é mostrada em uma maneira diferente na figura 6 , onde são mostrados pontos de dados da tensão de atrito e da pressão normal atuando nas placas laterais para as dimensões da placa $(260 \times 1900 \mathrm{~mm}, 210 \times 1225 \mathrm{~mm}$ e $260 \mathrm{x}$ $1600 \mathrm{~mm}$ ) e tipos de aço diferentes (aço peritético, aço de baixo e alto teor de carbono). Ambos os valores mostram uma grande variação nos seus dados, mas a 
aplicação da equação (5), o cálculo do coeficiente de atrito, mostra que o coeficiente de atrito tem uma variação muito menor.

(a)

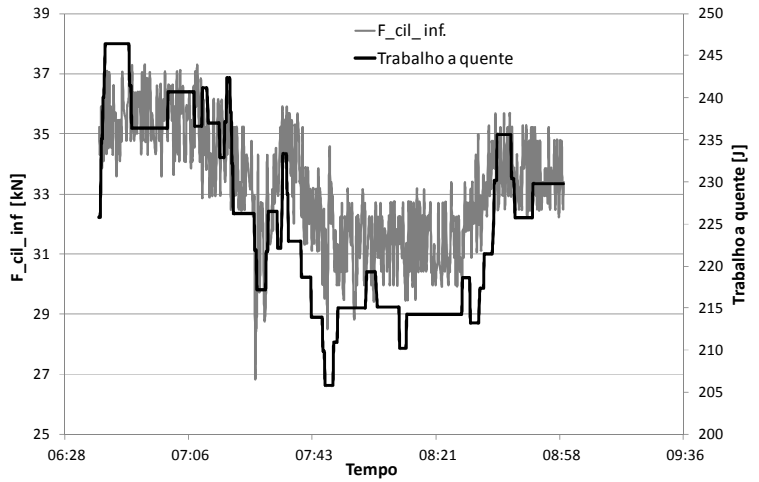

(b)

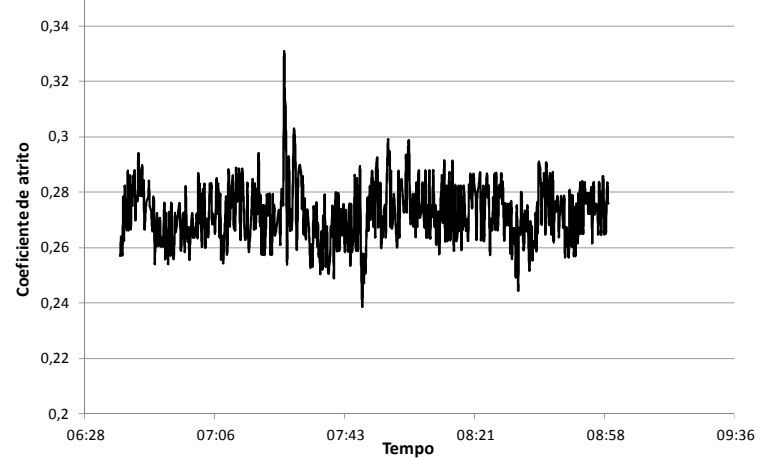

Figura 5. Força do cilindro, trabalho a quente e coeficiente de atrito em função do tempo.

A figura 7 mostra o comportamento do coeficiente de atrito com a velocidade de lingotamento durante uma sequência de lingotamento. Neste exemplo, o tipo de aço e as dimensões da placa foram mantidos constantes. Observa-se que entre 0,5 e $1,2 \mathrm{~m} / \mathrm{min}$, o coeficiente de atrito não mostra uma grande variação nos pontos de dados e ficou num valor médio de 0,27. Apenas nos casos onde a velocidade de lingotamento estava menor do que $0,5 \mathrm{~m} / \mathrm{min}$ (incluindo os casos onde a velocidade foi zerada), e as placas laterais perderam provavelmente o contato com o veio, os valores mostram uma variação grande.

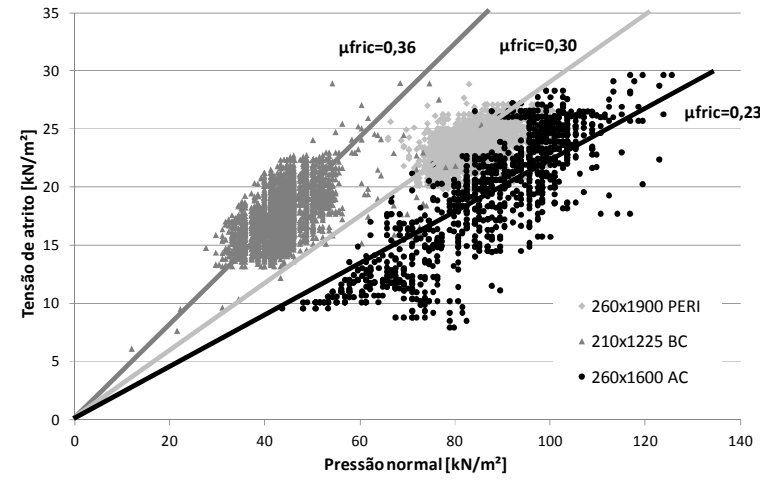

Figura 6. Tensão de atrito e pressão normal para vários tipos de aço e dimensões da placa.

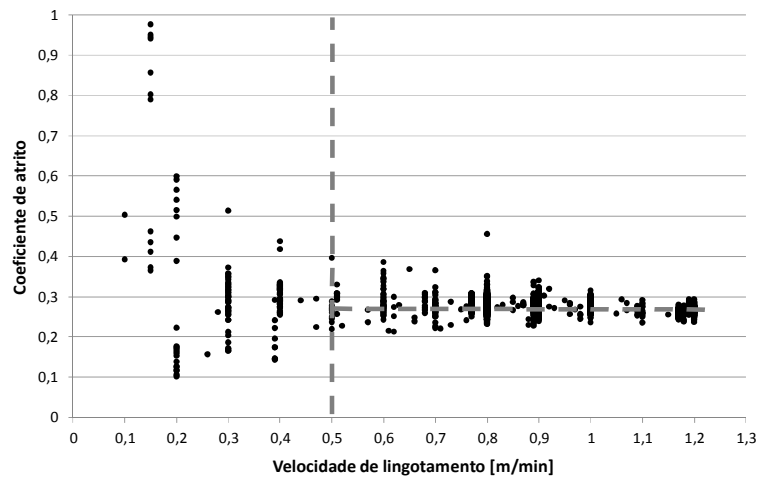

Figura 7. Coeficiente de atrito em função da velocidade de lingotamento.

Demonstrado que o coeficiente de atrito é um valor adequado para a avaliação da lubrificação entre as placas de molde e o veio, duas aplicações práticas serão mostradas em seguida.

A primeira aplicação é o ajuste da prática de oscilação (curso e frequência em função da velocidade de lingotamento). A figura 8 mostra a mudança do coeficiente de atrito com a mudança da prática de oscilação. A velocidade de lingotamento foi mantida constante. Neste exemplo, a segunda prática oscilação com um curso maior e uma frequência menor mostrou uma redução instantânea do coeficiente de atrito de 0,27 para 0,22 .

O coeficiente de atrito do molde é também um parâmetro confiável para auxiliar no desenvolvimento de pó fluxante. A figura 9 mostra a mudança do coeficiente de atrito durante o teste de três pós fluxante diferentes enquanto a prática de oscilação 
foi mantida constante. O terceiro pó aplicado mostrou uma redução do coeficiente de atrito de 0,23 para 0,21.

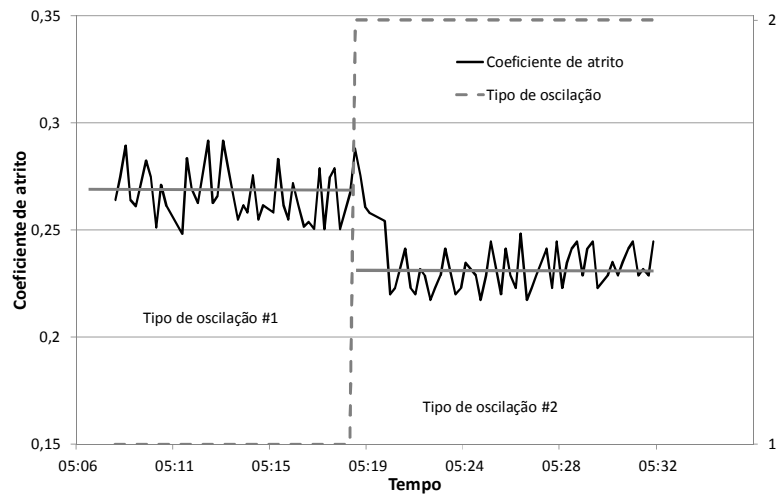

Figura 8. Influência da prática da oscilação ao coeficiente de atrito.

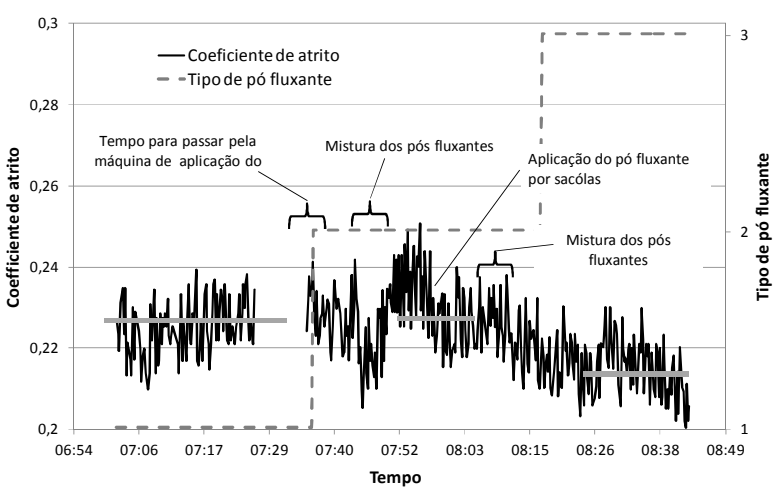

Figura 9. Influência do tipo de pó fluxante ao coeficiente de atrito.

\section{CONCLUSÃO}

No presente trabalho, uma metodologia para determinar o coeficiente de atrito entre as placas do molde e o veio foi desenvolvida. A determinação desse coeficiente baseia-se na combinação da medição da tensão de atrito e das forças atuando nas placas laterais do molde. O controle em tempo real do coeficiente de atrito auxilia no desenvolvimento de pó fluxante e na otimização de parâmetros de oscilação e possibilita um maior controle da qualidade da placa e do processo do lingotamento.

\section{REFERÊNCIAS}

1 Wolf MM. Review of Mould Friction. Seminar of Professor Brian Thomas; Leoben, Austria: Wolftechnology; 2000.

2 Meng Y, Thomas B. Modeling Transient Slag-Layer Phenomena in the Shell/mold Gap in Continuous Casting of Steel. Metallurgical and Materials Transactions B. 2003; 34: 707-725.

3 Mörwald K, Steinrück H, Rudischer C. Theoretical Studies to Adjust Proper Mould Oscillation Parameters. AISE Conference Proc.; Chicago, IL, USA: AISE; 2000.

4 Royzman SE. Coefficient of friction between strand and mould during continuous casting: mathematical model, Ironmaking and Steelmaking. 1997; 24(6):484-488.

5 Suzuki M, Mizukami H, Kitagawa T, Kawakami K, Uchida S, Komatsu Y. Development of a New Mold Oscillation Mode for High-speed Continuous Casting of Steel Slabs. ISIJ International. 1991; 31(3): 254-261.

6 Tsutsumi K, Ohtake J, Hino M. Inflow behavior observation of molten mold powder between mold and solidified shell by continuous casting simulator using $\mathrm{Sn}-\mathrm{Pb}$ alloy and stearic acid. ISIJ International. 2000; 40(6): 601-608.

7 Nakato H, Omiya S, Habu Y, Hamagami K, Koshikawa T. Optimizing Mold Lubrication for High-Speed Continuous Casting of Slabs. Journal of metals. 1984; 36(3): 44-50.

8 Wolf MM. Mould oscillation guidelines. Steelmaking Conference Proc.; Warrendale, PA, USA: Iron \& Steel Soc.; 1991: 51-67.

9 Lang O, Federspiel C, Thalhammer M, Watzinger J. The MoldEXPERT by VAI - A Mold Monitoring System for best Casting Performance. VAl 8th Continuous Casting Conference Proc.; Linz, Austria: VAl Industrieanlagenbau; 2000: 16.1-16.7.

10 Yao M, Fang DC. Online measuring method for mold friction in continuous-casting. Ironmaking and Steelmaking. 1996; 23(6): 522-527.

11 Duncan D. History of Tribology, $2^{\text {nd }}$ Edition. London: Professional Engineering Publishing; 1998. 